\title{
Caracterização física e determinação do teor de extrativos da madeira de Embiratanha
}

Francisco Edislan Gurgel Diógenes ${ }^{1}$, Juliana Holanda Maia ${ }^{1}$, Lohany Sthefany Souza ${ }^{1}$, Tallyta Martins de Sousa ${ }^{1}$, Vinicius Gomes de Castro ${ }^{*}$

\author{
${ }^{1}$ Universidade Federal Rural do Semi-Árido, R. Francisco Mota, 572 - Pres. Costa e Silva, Mossoró - RN, 59625-900 \\ *Author for correspondence: vinicius.castro@ ufersa.edu.br
}

Received: May 2019/ Accepted: September 2019/ Published: September 2019

\section{Resumo}

O objetivo deste trabalho foi caracterizar a madeira da espécie Pseudobombax marginatum (Embiratanha) a diferentes alturas do tronco quanto a suas propriedades físicas e ainda comparar os teores de extrativos destas amostras de madeiras com a casca da espécie. Três indivíduos foram coletados em uma região semiárida do estado do Rio Grande do Norte e seccionados em cinco diferentes alturas. A casca foi separada independente da altura. Foram avaliadas as massas específicas (aparente e básica) e os índices de contração lineares e volumétrico da madeira, além dos teores de extrativos polares e apolares da madeira e da casca. Não foi observada diferença estatística entre as médias das propriedades físicas avaliadas para as madeiras de diferentes alturas do tronco. A madeira foi considerada leve, porém com uma boa estabilidade dimensional, apresentando valores de contração baixos e coeficiente de anisotropia abaixo de 1,5. Também não foi encontrada diferença entre os teores de extrativos polares da madeira e da casca da espécie. Porém, a quantidade de extrativos polares foi superior na casca, sendo que ainda pode ser observada uma quantidade inferior deste tipo de extrativo na região central do tronco em comparação com a base ou o topo. Com base nos resultados, a madeira de Embiratanha mostrou-se uma alternativa como produto substituto para outras madeiras de baixa massa específica mais tradicionais no mercado.

Palavras-chave: Caatinga; Baixa densidade; Coeficiente de anisotropia

\footnotetext{
Abstract

The aim of this work was characterize the Pseudobombax marginatum (Embiratanha) wood from different trunk heights according to its physical properties and yet to compare the extractives amounts between wood samples and the species bark. Three individuals were collected in a semiarid region of Rio Grande do Norte state and were cut into five different heights. The bark was segregated independent from the height. The specific gravities (apparent and basic) and the linear and volumetric shrinkage index of the wood were evaluated, as well as the polar and apolar extractives amount of wood and bark. There was no statistic difference among the avereges of physical properties of wood from different trunk heights. The wood was considered light, however with good dimensional stability, showing shrinkage values low and anisotropy coefficient lower than 1.5. There was also no difference between polar extractive of wood and bark. However, the apolar extractive amount was higher in the bark, and was also observed that the wood from the middle part of the trunk showed lower amount than the stump or the top. Based on the results, the Embiratanha wood presented itself as an alternative product for others traditional low density wood that can be found in the market.
}

Keywords: Caatinga, Low density, Anisotropic coefficient

\section{Introdução}

A espécie Pseudobombax marginatum (A.St.-Hil., Juss. \& Cambess.) A. Robyns, da família Malvaceae, é, geralmente, conhecida pelo nome comum Embiratanha, sendo que na região Nordeste, também pode ser chamada de Imbiriçu ou Imbiratanha, onde ocorre nos estados da Bahia, Ceará, Maranhão, Paraíba, Pernambuco, Piauí e Rio Grande do Norte (Flora do Brasil 2019). Porém, ela não é uma espécie endêmica do Brasil, embora tenha a distribuição exclusiva na América do Sul, sendo também encontrada especialmente na Bolívia, Paraguai e Peru (Bocage e Sales 2002).

A madeira de Embiratanha é considerada leve, sendo relatados na literatura valores médios de massa específica básica entre 0,26 e $0,55 \mathrm{~g} / \mathrm{cm} 3$ para indivíduos na região semiárida do Brasil (Lima et al. 2012; Silva et al. 2014; Fagundes e Weisser 2018). Devido a esta característica, sua madeira é tradicionalmente limitada a usos que exige baixa resistência mecânica, como por exemplo: confecção de caixotes, forro, brinquedos e calçados (Campanha e Araújo 2010).

Lucena et al. (2012) realizaram um levantamento em comunidades rurais da Paraíba e indicaram que a madeira desta espécie não foi apontada como potencial para produção de energia, construção ou para tecnologia, sendo a planta geralmente usada apenas para fins medicinais ou veterinários. $\mathrm{Na}$ região da caatinga potiguar, a casca é popularmente usada em decocção ou em forma de chá como anti-inflamatória para úlceras, gastrite ou dores nas costas, propriedade medicinal esta que já foi cientificamente comprovada (Paiva et al. 2013). Sendo que uso terapêutico da Embiratanha já foi remetido a humanos pré-históricos da região de Furna do Estrago, Pernambuco (Texeira-Santos et al. 2015). As propriedades anti-microbiais da casca desta espécie estão ligadas aos teores de extrativos polifenóis e flavonóides que variam de acordo com o período de coleta, apresentando maior concentração em épocas de chuva (Chaves et al. 2013)

Contudo, embora haja um relativo interesse no estudo e caracterização das cascas da Embiratanha, pouca pesquisa é voltada para a madeira desta espécie. O primeiro passo na busca de um uso adequado para este material seria sua caracterização. Desta forma, objetivou-se com a presente pesquisa, caracterizar fisicamente a madeira, e quimicamente a madeira e a casca da espécie Pseudobombax marginatum ao longo de seu tronco.

\section{Material e Métodos}

Foram coletados três indivíduos de Pseudobombax marginatum (A.St.-Hil., Juss. \& Cambess.) A. Robyns (Embiratanha) em uma área manejada localizada no município de Upanema, RN. Os troncos de cada indivíduo 
foram seccionados em cinco discos de aproximadamente 5 $\mathrm{cm}$ de espessura nas alturas a 0 (base), 25, 50, 75 e $100 \%$ (topo) em relação à altura comercial.

Para cada disco, foram produzidos corpos de prova de aproximadamente $2,5 \mathrm{~cm}^{2}$ de área transversal e $5 \mathrm{~cm}$ de comprimento longitudinal. Foram obtidas as medidas das dimensões, por meio de um paquímetro, e massa, através de balança de precisão, dos corpos de prova secos, em teor de umidade de equilíbrio a 12\% (TUE) e em ponto de saturação das fibras (PSF). Com estes dados, foram então calculadas as massas específicas (aparente e básica) e os índices de contração lineares e volumétrico (Tabela 1) das madeiras em diferentes alturas do tronco de acordo com a norma NBR 7190 (ABNT 1997).

Tabela 1. Fórmulas utilizadas na caracterização física da madeira de Pseudobombax marginatum em diferentes alturas do tronco

\begin{tabular}{|c|c|}
\hline $\begin{array}{l}\text { Massa específica } \\
\text { aparente }\left(\mathrm{g} / \mathrm{cm}^{3}\right)\end{array}$ & $\rho_{\mathrm{zp}}=\frac{\mathrm{m}_{12}}{\mathrm{v}_{12}}$ \\
\hline $\begin{array}{c}\text { Massa específica básica } \\
\left(\mathrm{g} / \mathrm{cm}^{3}\right)\end{array}$ & $\rho_{\mathrm{bas}}=\frac{\mathrm{m}_{\mathrm{s}}}{\mathrm{v}_{\mathrm{sat}}}$ \\
\hline $\begin{array}{c}\text { Contração Volumétrica } \\
(\%)\end{array}$ & $\beta V_{\max }=\frac{v_{s a t}-v_{5}}{v_{s a t}}$ \\
\hline $\begin{array}{c}\text { Contração Tangencial, } \\
\text { Radial e Longitudinal } \\
(\%)\end{array}$ & $\beta \mathbf{T}_{v} \mathbf{R}, \mathbf{L}=\frac{\mathbf{T}_{v} \mathbf{R}_{v} \mathbf{L}_{s 2 \mathrm{t}}-\mathbf{T}_{v} \mathbf{R}_{v} \mathbf{L}_{s}}{\mathbf{T}_{v} \mathbf{R}_{v} \mathbf{L}_{s 2 \mathrm{t}}}$ \\
\hline $\begin{array}{l}\text { Coeficiente de } \\
\text { Anisotropia }\end{array}$ & $\mathrm{CA}=\frac{\beta \text { Tangencial }}{\beta \text { Radial }}$ \\
\hline
\end{tabular}

Sendo: $P_{\text {ap }}=$ massa específica aparente; $\mathbf{m}_{12}=$ massa em teor de umidade em equilíbrio a $12 \%$; $\mathbf{v}_{12}=$ volume em teor de umidade em equilíbrio a $12 \%$; $P_{\text {bas }}=$ massa específica básica; $\mathbf{m}_{\mathbf{s}}=$ massa seca; $\mathbf{v}_{\text {sat }}=$ volume saturado; $\beta \mathrm{V} \max$ $=$ contração volumétrica; $\mathbf{v}_{\boldsymbol{S}}=$ volume $\operatorname{seco} ; \beta \mathbf{T}_{v} \mathbf{R}, \mathbf{L}=$ Contração Tangencial, Radial e Longitudinal; $\mathbf{T}_{v} \mathbf{R}, \mathbf{L}_{s a t}=$ medida Tangencial, Radial e Longitudinal saturada; $\mathbf{T}_{v} \mathbf{R}, \mathbf{L}_{\mathbf{s}}$ $=$ medida Tangencial, Radial e Longitudinal seca; $\mathrm{CA}=$ Coeficiente de Anisotropia; $\beta$ Tangencial = Contração Tangencial; $\beta$ Radial = Contração Radial.

Parte dos discos excedentes da produção dos corpos de prova para análise das propriedades físicas foram moídas em um moinho de facas tipo Wiley e peneiradas para análise do teor de extrativos da espécie em diferentes alturas. Para as análises, foi utilizada o material passante em peneira de 40 mesh e retido em peneira de 100 mesh. As cascas dos discos amostrados foram agrupadas em um único lote e submetidos ao mesmo processo de preparação para análise química do que as madeiras. Para cada análise química, foram utilizadas 3 repetições referentes a cada altura e casca. Com isso, foram determinados os teores de extrativos polares em água quente (TAPPI 1999) e o teores de extrativos apolares, utilizando acetona como solvente orgânico (TAPPI 1997). Os extrativos totais foram determinados pela soma dos extrativos polares e apolares.

Os dados obtidos das propriedades físicas e químicas observadas foram submetidos à análise de variância (ANOVA) a 5\% de significância, sendo que, preliminarmente, a normalidade dos dados foi caracterizada pelos testes Skewness e Kurtosis. Nos casos de rejeição da hipótese da nulidade, aplicou-se o teste Tukey, em nível de probabilidade de $95 \%$, para diferenciação das médias. Foi ainda aplicado um modelo de regressão linear simples para analisar a relação entre massa específica básica da espécie e sua contração volumétrica, considerando todas as amostras, independente da altura do tronco da qual foram retiradas.

\section{Resultados}

Não houve diferença estatística entre os valores médios de todas as propriedades físicas avaliadas para os corpos de prova retirados em diferentes alturas do tronco da Embiratanha (Tabela 2). Desta forma, os valores médios das propriedades podem ser considerados representativos do comportamento da madeira da espécie.

Tabela 2. Caracterização física da madeira de Pseudobombax marginatum em diferentes alturas do tronco

\begin{tabular}{|c|c|c|c|c|c|c|}
\hline \multirow{2}{*}{ Propriedades Físicas } & \multicolumn{5}{|c|}{ Altura em relação ao tronco } & \multirow{2}{*}{ Média } \\
\hline & Base & $25 \%$ & $\mathbf{5 0} \%$ & $75 \%$ & Topo & \\
\hline \multirow{2}{*}{ ME aparente $\left(\mathrm{g} / \mathrm{cm}^{3}\right)$} & $0,31^{a}$ & $0,30^{\mathrm{a}}$ & $0,32^{\mathrm{a}}$ & $0,34^{a}$ & $0,38^{\mathrm{a}}$ & 0,33 \\
\hline & $(22,40)$ & $(15,03)$ & $(20,25)$ & $(21,83)$ & $(18,27)$ & $(20,35)$ \\
\hline \multirow{2}{*}{ ME básica $\left(\mathbf{g} / \mathrm{cm}^{3}\right)$} & $0,23^{\mathrm{a}}$ & $0,24^{\mathrm{a}}$ & $0,26^{\mathrm{a}}$ & $0,27^{\mathrm{a}}$ & $0,31^{a}$ & 0,26 \\
\hline & $(25,52)$ & $(17,04)$ & $(17,84)$ & $(21,66)$ & $(17,27)$ & $(21,60)$ \\
\hline \multirow{2}{*}{ Contração Volumétrica (\%) } & $11,31^{a}$ & $10,41^{\mathrm{a}}$ & $8,36^{\mathrm{a}}$ & $9,72^{\mathrm{a}}$ & $7,03^{\mathrm{a}}$ & 9,53 \\
\hline & $(34,49)$ & $(21,38)$ & $(29,56)$ & $(23,19)$ & $(43,80)$ & $(31,42)$ \\
\hline \multirow{2}{*}{ Contração Tangencial (\%) } & $4,28^{\mathrm{a}}$ & $4,03^{\mathrm{a}}$ & $4,18^{\mathrm{a}}$ & $3,91^{\mathrm{a}}$ & $3,51^{\mathrm{a}}$ & 3,97 \\
\hline & $(31,88)$ & $(23,03)$ & $(20,25)$ & $(34,50)$ & $(15,61)$ & $(25,23)$ \\
\hline \multirow{2}{*}{ Contração Radial (\%) } & $4,05^{\mathrm{a}}$ & $2,53^{\mathrm{a}}$ & $2,61^{\mathrm{a}}$ & $3,43^{\mathrm{a}}$ & $3,21^{a}$ & 3,15 \\
\hline & $(20,42)$ & $(32,33)$ & $(19,15)$ & $(38,13)$ & $(21,14)$ & $(31,22)$ \\
\hline \multirow{2}{*}{ Contração Longitudinal (\%) } & $0,93^{a}$ & $0,48^{\mathrm{a}}$ & $0,79^{\mathrm{a}}$ & $0,68^{\mathrm{a}}$ & $0,63^{\mathrm{a}}$ & 0,71 \\
\hline & $(60,02)$ & $(63,22)$ & $(62,79)$ & $(55,03)$ & $(62,79)$ & $(59,70)$ \\
\hline \multirow{2}{*}{ Coeficiente de Anisotropia } & $1,25^{\mathrm{a}}$ & $1,75^{\mathrm{a}}$ & $1,65^{\mathrm{a}}$ & $1,17^{\mathrm{a}}$ & $1,16^{\mathrm{a}}$ & 1,39 \\
\hline & $(18,62)$ & $(38,39)$ & $(26,90)$ & $(24,55)$ & $(17,69)$ & $(31,74)$ \\
\hline
\end{tabular}

Sendo: ME, massa específica. Valores seguidos de mesma letra em uma mesma linha não apresentam diferença estatística significante a $5 \%$ de probabilidade. Valores em parênteses relativo ao Coeficiente de Variação (\%).

Os valores médios de massa específica básica e aparente foram abaixo dos valores mínimos exigidos para madeiras estruturais determinados pela norma brasileira, que é de 0,5 e $0,65 \mathrm{~g} / \mathrm{cm}^{3}$, respectivamente (ABNT 1997). De acordo com a classificação sugerida por Eleotério et al. (2015), a madeira desta espécie pode ser classificada como muito leve, por apresentar massa específica aparente abaixo de 0,4 $\mathrm{g} / \mathrm{cm}^{3}$.

A madeira de Embiratanha apresentou uma boa estabilidade dimensional, uma vez que os valores médios encontrados para contração volumétrica, tangencial e radial foram considerados baixos, sendo inferiores aos limites de $12,32 \%, 7,43 \%$ e $3,51 \%$, respectivamente (Eleotério et al. 2015). A estabilidade também foi confirmada pelo fato do Coeficiente de Anisotropia ter sido inferior a 1,5 (Klitzke et al. 2008). Contudo, não pode ser observada uma correlação linear forte entre a contração volumétrica das amostras e suas massas específicas (Figura 1). 


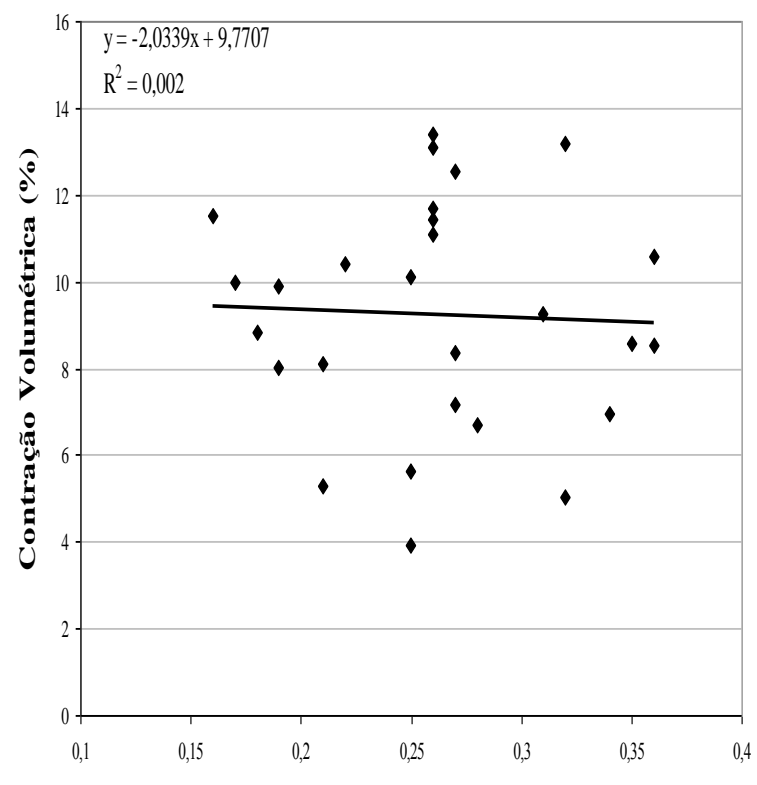

Massa específica básica $\left(\mathrm{g} / \mathrm{cm}^{3}\right)$

Figura 1. Correlação linear entre a massa específica básica e a contração volumétrica da espécie Pseudobombax marginatum.

Em relação à quantidade de extrativos presentes na madeira, não houve diferença significativa entre os valores médios dos extrativos solúveis em água quente em diferentes posições do tronco ou presentes na casca da Embiratanha (Figura 2a). Contudo, pode ser observado que em relação aos extrativos solúveis em acetona, a casca da madeira e nas proporções superiores do tronco (75\% e 100\% da altura do tronco comercial), além da base, apresentaram valores médios superiores às regiões centrais $(25 \%$ e $50 \%)$. Por sua vez, a quantidade de extrativos apolares na casca foi superior à encontrada na madeira independente da posição (Figura 2b).
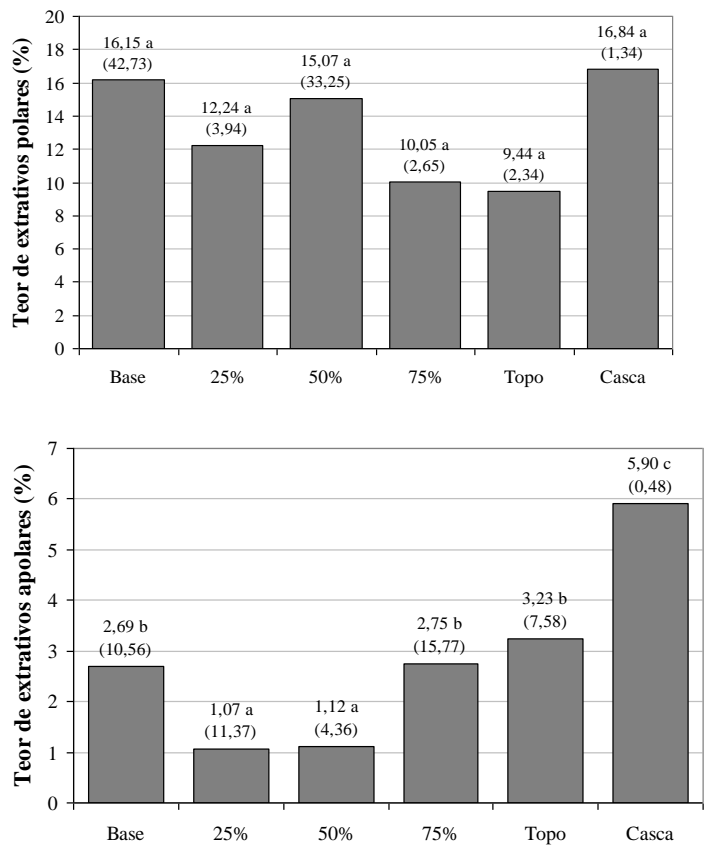

Figura 2. Teores de extrativos da madeira de Pseudobombax marginatum em diferentes alturas do tronco e da casca (Valores seguidos de mesma letra em um mesmo gráfico não apresentam diferença estatística significante a $5 \%$ de probabilidade. Valores em parênteses relativo ao Coeficiente de Variação (\%)).

A quantidade de extrativos totais da madeira variou entre $12,80 \%$ e $18,84 \%$ dependendo da posição do tronco. Estes valores indicam que a madeira de Embiratanha contém uma grande quantidade de extrativos, uma vez que a quantidade média de extrativos de madeira tropicais é de 7,6\% (Brémaud et al. 2011).

\section{Discussão}

O valor médio da massa específica básica da espécie estudada $\left(0,26 \mathrm{~g} / \mathrm{cm}^{3}\right)$, embora abaixo do valor relatado por Silva et al. (2014), já foi encontrado por outros pesquisadores em indivíduos da região semiárida de Pernambuco (Lima et al. 2012). A baixa massa específica da madeira de Embiratanha aproxima-se de outra madeira que possui um mercado de consumo já estabelecido: o Pau-Balsa (Ochroma pyramidale). A madeira de Pau-Balsa também possui baixa massa específica, que pode variar de 0,06 a $0,38 \mathrm{~g} / \mathrm{cm}^{3}$, e pode ser classificada comercialmente em três classes de massa específica: leve (até $0,12 \mathrm{~g} / \mathrm{cm}^{3}$ ); média (entre 0,12 e $0,18 \mathrm{~g} / \mathrm{cm}^{3}$ ); e pesada (acima de $0,18 \mathrm{~g} / \mathrm{cm}^{3}$ ) (Borrega et al. 2015). Painéis sarrafeados que utilizam a madeira de Pau-Balsa na parte interna são aplicados em indústrias navais, aeroespaciais, de energia eólica, entre outros (Kotlarewski et al. 2016). Com relação a massa específica básica, a madeira de Embiratanha, quando comparada com a madeira de Pau-Balsa, apresenta um valor médio semelhante à categoria pesada desta última, um indicativo inicial que a madeira da Caatinga poderia vir a ser um produto substituto potencial.

Contudo, a contração volumétrica da madeira de Embiratanha $(9,53 \%)$ foi superior à relatada por Lobão et al. (2011) para madeira de Pau-Balsa (6,27\%) de massa específica semelhante. A diferença entre as contrações de madeiras de mesma massa específica pode ser explicada pela diferença no ângulo microfibrilar da camada S2 de suas paredes celulares. Ângulos maiores acarretam em uma contração tangencial maior, e consequentemente, uma maior contração volumétrica (Schulgasser e Witztum 2015). Desta forma, ao mesmo tempo que a diferença anatômica entre espécies justifica a diferença na estabilidade, a falta de correlação linear entre as massas específicas e a contração volumétrica das amostras de Embiratanha (Figura 1) indica que pode não haver diferença significativa na variação do ângulo microfibrilar entre as diferentes alturas do tronco.

Por outro lado, o uso potencial da espécie Embiratanha é promissor, entre outras razões, pelo valor médio de coeficiente de anisotropia apresentado $(1,39)$. Este fator foi inferior ao de outras espécies de baixa massa específica relatados na literatura, como por exemplo: o Pau d'alho (Gallesia integrifolia), espécie que tem massa específica básica variando entre 0,26 e $0,41 \mathrm{~g} / \mathrm{cm}^{3}$ e coeficiente de anisotropia médio de 1,84 (Motta et al. 2014); ou ainda, a madeira da espécie Paulownia elongata, espécie asiática conhecida como Kiri Japonês, que apresenta massa específica de $0,24 \mathrm{~g} / \mathrm{cm}^{3}$ e coeficiente de 2,32 (Bao et al. 2001).

Do ponto de vista químico, a quantidade de extrativos polares varia ao longo da altura do tronco de um mesmo indivíduo, sendo maior na base e, frequentemente, maior no topo e galhos do que nas alturas medianas do tronco. Contudo a composição destes extrativos tende a mudar da base em comparação com o topo (Jansson e Nilvebrant 2009). Esta relação quantitativa de extrativos solúveis em acetona ao longo do tronco foi observada na espécie Embiratanha. Tanto os valores médios de extrativos apolares, quanto o comportamento em relação à altura do 
tronco, foram semelhantes aos relatados na literatura para a espécie Pau-Balsa, de massa específica semelhante. Honorato-Salazar et al. (2015) apresentaram um teor médio de extrativos solúveis em Etanol-Benzeno para a madeira de Pau-Balsa de $3,12 \%$, sendo que a parte central do tronco apresentou valor médio estatisticamente inferior as madeiras da base e do topo. Bao et al. (2001) também observaram que a madeira juvenil de Paulownia elongata apresentou uma maior quantidade de teor de extrativos solúveis em ÁlcoolBenzeno em relação a madeira madura $(8,81 \%$ e $8,24 \%$, respectivamente).

A quantidade de extrativos tende a ser maior na casca do que na madeira (Jansson e Nilvebrant 2009). Porém, a composição química dos extrativos da casca e da madeira são semelhantes, sendo que a principal diferença é que cascas de muitas espécies contém mais polifenóis e suberina de maior peso molecular (Feng et al. 2013). Isso pode ser observado na Embiratanha uma vez que não houve diferença estatística entre as médias dos extrativos polares da madeira e da casca, mas o teor de extrativos apolares foi estatisticamente superior na casca do que na madeira, independente da posição na altura do tronco.

\section{Conclusão}

A madeira de Pseudobombax marginatum (Embiratanha) é uma espécie de baixa massa específica, mas que possui características positivas que a torna uma boa candidata a ser uma potencial substituta de outras madeiras leves presentes no mercado. Além de não haver variação das propriedades físicas da madeira em diferentes alturas da árvore, que possibilita a exploração do tronco como um todo, ainda apresenta um coeficiente anisotrópico e valores de contração considerados baixos, que demonstra uma boa estabilidade dimensional.

\section{Referências}

ABNT - Associacão Brasileira de Normas Técnicas. NBR 7190: Projeto de estruturas de madeiras. São Paulo, 1997.

Bao FC, Jiang ZH, Jiang XM, Lu XX, Luo XQ, Zhang SY (2001) Differences in wood properties between juvenile wood and mature wood in 10 species grown in China. Wood Science and Technology, 35(4):363-375. doi: $10.1007 / \mathrm{s} 002260100099$

Bocage ALD, Sales MF (2002) A família Bombacaceae Kunth no estado de Pernambuco, Brasil. Acta Botanica Brasileira, 16(2): 123-139. doi: 10.1590/S010233062002000200001

Borrega M, Ahvenainen P, Serimaa R, Gibson L (2015) Composition and structure of balsa (Ochroma pyramidale) wood. Wood Science and Technology, 49(2): 403-420. doi: $10.1007 / \mathrm{s} 00226-015-0700-5$

Brémaud I. Amusant H. Minato K. Gril J. Thibaut B (2011) Effect of extractives on vibrational properties of African Padauk (Pterocarpus soyauxii Taub.). Wood Science and Technology, 45(3): 461-472. doi: 10.1007/s00226-0100337-3

Campanha MM. Araúio FS (2010) Árvores e arbustos do sistema agrossilvipastoril: caprinos e ovinos. Sobral Embrapa Caprinos e Ovinos. 32p.

Chaves TP, Santana CP, Véras G, Brandão DO, Felismino DC, Medeiros ACD, Trovão DMBM (2013) Seasonal variationin the production of secondary metabolites and antimicrobial activity of two plant species used in
Brazilian traditional medicine. African Journal of Biotechnology, 12(8): 847-853. doi: 10.5897/AJB12.2579

Eleotério JR. Reichert D. Hornburg KF. Meneguelli I (2015) Massa específica e retratibilidade da madeira de seis espécies de Eucalipto cultivadas no litoral de Santa Catarina. Floresta, 45(2): 329-336. doi: 10.5380/rf.v45i2.34699.

Fagundes M. Weisser W (2018) The role of nurse successional stages on species-specific facilitation in drylands: Nurse traits and facilitation skills. Ecology and Evolution, 8(10): 5173-5184. doi: 10.1002/ece3.3962

Feng S, Cheng S, Yuan Z, Leitch M, Xu C (2013) Valorization of bark for chemicals and materials: a review. Renewable and Sustainable Energy Reviews, 26: 560-578. doi: 10.1016/j.rser.2013.06.024

Flora do Brasil (2019) Flora do Brasil 2020 em construção. Jardim Botânico do Rio de Janeiro.Disponível em: $<$ http://floradobrasil.ibri.gov.br/reflora/floradobrasil/FB25 762>. Acesso em: 29 Mar. 2019

Honorato-Salazar JÁ, Colotl-Hernández G, ApolinarHidalgo F, Aburto J (2015) Principales componentes químicos de la madeira de Ceiba pentandra, Hevea brasiliensis v Ochroma pvramidale. Maderas v Bosques, 21(2):131-146. doi: 10.21829/myb.2015.212450.

Jansson MB, Nilvebrant NO (2009) Wood extractives. In: Ek M, Gellerstedt G. Pulp and paper chemistry and technologv: wood chemistrv and biotechnology. Volume 1. Stockholm: De Gruyter. p.147-171

Klitzke RJ, Savioli DL, Muniz GIB, Batista DC (2008) Caracterização dos lenhos de cerne, alburno e transição de jatobá (Hvmenae sp.) visando ao agrupamento para fins de secagem convencional. Scientia Forestalis, 36(80): 279284.

Kotlarewski NJ, Belleville B, Gusamo BK, Ozarska B (2016) Mechanical properties of Papua New Guinea balsa wood. European Journal of Wood Products, 74: 83-89. doi: 10.1007/s00107-015-0983-0

Lima ALA, Sampaio, EVSB, Castro CC, Rodal MJN, Antonino ACD, Melo AL (2012) Do the phenology and functional stem attributes of woody species allow for the identification of functional groups in the semiarid region of Brazil? Trees, 26:1605-1616. doi: 10.1007/s00468-012$0735-2$

Lobão MS, Castro VR, Rangel A, Sarto C, Tomazello Filho M, Silva Júnior FG, Camargo Neto L, Bermudez MARC (2011) Agrupamento de espécies florestais por análises univariadas e multivariadas das características anatômica, física e química das suas madeiras. Scientia Forestalis, 39(92):469-477.

Lucena RFP, Medeiros PM, Araúio EL, Alves AGC, Albuaueraue UP (2012) The ecological apvarencv hypothesis and the importance of useful plants in rural communities from Northeastern Brazil: na assessment based on use value. Journal of Environmental Management, 96:106-115. doi: 10.1016/j.jenvman.2011.09.001

Motta JP, Oliveira, JTS, Braz RL, Duarte APC, Alves RC (2014) Caracterização da madeira de quatro espécies 
florestais. Ciência Rural. 44(12):2186-2192. doi: $10.1590 / 0103-8478 \mathrm{cr} 20130479$

Paiva DCC, Santos CA, Diniz JC, Viana FA, Thomazzi SM, Falcão DA (2013) Anti-inflammatory and antinociceptive effects of hydroalcoholic extract from Pseudobombax marginatum inner bark form caatinga potiguar. Journal of Ethnopharmacology, 149:416-421. doi: 10.1016/j.jep.2013.06.012

Silva AML, Lopes SF, Vitorio LAP, Santiago RR, Mattos EA, Trovão, DMBM (2014) Plant functional groups of species in semiarid ecosvstems in Brazil: wood basic density and SLA as an ecological indicator. Brazilian Journal of Botany, 37(3):229-237. doi: 10.1007/s40415014-0063-4

Schulgasser K, Witztum A (2015) How the relationship between densitv and shrinkage of wood depends on its microstructure. Wood Science and Technology, 49(2):389-401. doi: 10.1007/s00226-015-0699-7

TAPPI - Technical Association of the Pulp and Paper Industrv. TAPPI T $204 \mathrm{~cm}-97$. Solvent extractives of wood and pulp. Atlanta: Tappi Technology Park, 1997.

TAPPI - Technical Association of the Pulp and Paper Industry. TAPPI T $207 \mathrm{~cm}-99$. Water solubility of wood and pulp. Atlanta: Tappi Technology Park, 1999.

Texeira-Santos I, Sianto L, Araúio A, Reinhard KJ, Chaves SAM (2015) The evidence of medicinal plants in human sediments from Fauna do Estrago prehistoric site. Pernambuco State, Brazil. Quaternary International, 377(7):112-117. doi: 10.1016/j.quaint.2015.01.019. 